\title{
Atenção Primária e saúde materno-infantil: a percepção de cuidadores em uma comunidade rural quilombola
}

\author{
Primary Care and maternal and child health: \\ perceptions of caregivers in a rural 'quilombola' community
}

Amaro Sérgio Marques ${ }^{1}$

Daniel Antunes Freitas ${ }^{1}$

Cláudia Danyella Alves Leão ${ }^{1}$

Stéphany Ketllin Mendes Oliveira ${ }^{1}$

Mayane Moura Pereira ${ }^{1}$

Antônio Prates Caldeira ${ }^{1}$

${ }^{1}$ Departamento de Saúde da Mulher e da Criança, Universidade Estadual de Montes Claros. Campus Universitário Professor Darcy Ribeiro. 39.401-089 Montes Claros MG Brasil. amarosergiomarques@ gmail.com
Abstract This study sought to evaluate the attributes of primary care, with a focus on child health, from the viewpoint of a 'quilombola' community in the north of the state of Minas Gerais, Brazil (A quilombola is a resident of a quilombo in Brazil. They are the descendents of Afro-Brazilian slaves who escaped from slave plantations that existed in Brazil until abolition in 1888). It is a cross-sectional, descriptive study with a quantitative approach. The Primary Care Assessment Tool (PCA-Tool, child version) was used and applied to the main caregivers of child residents duly registered in the quilombola community. The attributes of primary care were assessed using scores awarded by caregivers, according to the criteria of the instrument. Only two attributes of primary care revealed satisfactory values: Access-Use and Information Coordination-System. The lowest scores were for the attributes of Family Guidance and Access-accessibility. The overall score was also below the satisfactory assessment. The results register low fidelity to attributes of primary care, as perceived by caregivers, and highlight the need for greater efforts to adapt the new model of care for the population under scrutiny.

Key words Primary Health Care, Family health, Child health, Ethnicity and health
Resumo Este estudo objetivou avaliar os atributos da atenção primária, com enfoque sobre a saúde infantil, segundo a percepção de uma comunidade quilombola no Norte de Minas Gerais. Tratase de pesquisa transversal, descritiva, de abordagem quantitativa. Utilizou-se o Instrumento de Avaliação da Atenção Primária, versão infantil (PCA-Tool, versão criança), com aplicação aos cuidadores das crianças residentes e cadastradas na comunidade quilombola. Os atributos da atenção primária foram avaliados através dos escores aferidos pelos cuidadores, segundo critérios do instrumento. Apenas dois atributos da atenção primária apresentaram valores satisfatórios: AcessoUtilização e Coordenação-Sistema de informação. Os piores escores foram para os atributos de Orientação Familiar e Acesso-acessibilidade. $O$ escore geral também mostrou valor abaixo do satisfatório. Os resultados registram baixa fidelidade aos atributos da atenção primária, segundo a percepção dos cuidadores e destacam a necessidade de maiores esforços para adequação do novo modelo assistencial para a população estudada.

Palavras-chave Atenção Primária à Saúde, Saúde da Família, Saúde da criança, Etnia e saúde 
Introdução

Ao longo de séculos a população negra formada por grupos remanescentes de escravos africanos no Brasil, geralmente organizada em comunidades rurais, foi segregada e teve seus direitos sociais negados. Reconhecidos como remanescentes de quilombos ou quilombolas, esse grupo social apenas nos últimos anos tem recebido atenção mais destacada das entidades governamentais. Políticas específicas na área da saúde também chegaram tardiamente para essa populaçãoํ.

No âmbito da assistência proposta pelo Sistema Único de Saúde (SUS), o atendimento prioritário às necessidades dessa população (entre outras) representa o princípio da equidade. Tal princípio não possui um conceito simples, mas na área da saúde tem sua expressão na redução máxima das desigualdades e diferenças evitáveis e injustas ${ }^{2}$. Para Coelho, ainda persistem muitas desigualdades no processo assistencial de saúde, incluindo-se discrepâncias entre as regiões urbanas e rurais ${ }^{3}$. Estudos avaliativos que busquem aferir a efetividade e o alcance das políticas de saúde junto aos grupos mais vulneráveis da população brasileira, incluindo-se aí as comunidades negras e quilombolas, representam um compromisso social e um apoio na construção da equidade.

Nos últimos anos, a transformação desejada para a atenção à saúde no país busca o fortalecimento da Atenção Primária, o desenvolvimento de ações de saúde com ênfase na promoção e ampliação da assistência ambulatorial, de forma descentralizada ${ }^{4}$. A Estratégia de Saúde da Família (ESF), eixo central da política de Atenção Primária à Saúde (APS) no país tem o potencial de interferir favoravelmente sobre indicadores de saúde da população, inclusive reduzindo internações por condições sensíveis ao cuidado primário ${ }^{5,6}$.

É importante reconhecer, entretanto, que o aumento apenas numérico de equipes de saúde não implica em mudança de modelo e pode perpetuar, de forma velada, a situação de exclusão e abandono de determinados grupos étnico-raciais, o que reitera a ideia do racismo institucional ${ }^{7}$. Para uma mudança efetiva, o novo modelo de atenção à saúde deve incorporar valores, princípios e elementos próprios (atributos) da APS. Alguns estudos demonstram que os sistemas de serviços de saúde que se organizam a partir de uma APS estruturada em conformidade com os seus atributos ordenadores, são mais eficazes e possuem maior qualidade ${ }^{5,8,9}$.
Starfield definiu quatro atributos essenciais da APS (primeiro contato, continuidade ou longitudinalidade, integralidade e coordenação) e dois atributos derivados (centralização familiar e orientação comunitária) ${ }^{8}$. A percepção dos diferentes atores envolvidos com a atenção à saúde permite identificar, por meio de instrumentos validados, a presença e a extensão dos atributos da APS para o serviço assistido. Alguns estudos nacionais já destacaram que as equipes da ESF ainda não apresentam total aderência aos atributos definidos por Starfield ${ }^{10-12}$. Todavia, ainda não existem estudos voltados à percepção de grupos historicamente excluídos. Este aspecto é particularmente importante, pois destaca o quanto e como as entidades governamentais estão atuando para o enfrentamento das iniquidades em saúde. Assim, o presente estudo teve como objetivo avaliar os atributos da atenção primária, com enfoque sobre a saúde infantil, segundo a percepção de uma comunidade quilombola no Norte de Minas Gerais.

\section{Metodologia}

Trata-se de um estudo transversal, descritivo, analítico, de abordagem quantitativa. $\mathrm{O}$ cenário deste estudo foi a comunidade rural quilombola de Buriti do Meio, remanescente de quilombo, situada no Distrito de Vila do Morro, município de São Francisco ao norte do estado de Minas Gerais. A comunidade é certificada pela Fundação Palmares e localiza-se a cerca de $30 \mathrm{~km}$ do município sede. A principal fonte de renda dessas famílias provém de atividades agrícolas, de benefícios sociais e da prestação de serviços aos fazendeiros da região. Outro destaque é dado ao artesanato de argila que mobiliza parte da comunidade.

A população alvo do presente estudo foi constituída pelos cuidadores das crianças de zero a cinco anos de idade, residentes e cadastradas nas áreas de abrangência da equipe da ESF Lapa Grande, na referida comunidade. Não houve seleção amostral, pois o interesse da proposta foi atuar com o universo materno-infantil da comunidade. Nos domicílios onde havia mais de uma criança na faixa etária definida o questionário foi aplicado com dados referentes à criança mais nova.

Para coleta de dados foi utilizado um instrumento já validado no Brasil, chamado Instrumento de Avaliação da Atenção Primária (PCATool - do inglês Primary Care Assessment Tool), cujo objetivo é medir a extensão dos atributos da 
APS em serviços de atenção à saúde infantil. O instrumento é composto por 55 ítens divididos em 10 componentes, que incorporam além dos atributos da APS, o 'Grau de Afiliação com Serviço de Saúde’ e foi recentemente assumido pelo Ministério da Saúde ${ }^{13}$. Para aplicação das questões, utiliza-se uma escala tipo Likert, constando de assertivas relacionadas a cada um dos atributos avaliados com o objetivo de verificar o nível de concordância/discordância do entrevistado (cuidador da criança).

Também foram coletados dados sobre características demográficas, socioeconômicas das famílias selecionadas. A entrevista foi realizada com o cuidador principal das crianças identificadas nos seus próprios domicílios e foi realizada por estudantes da área da saúde, especialmente treinados. A coleta foi realizada entre os meses de outubro e dezembro de 2011.

Os dados foram processados por meio do pacote estatístico SPSS for windows (versão 18.0). Para aferição da qualidade da assistência na APS foram calculados os escores de cada atributo (que são obtidos pela média dos itens que compõe cada atributo) e o escore geral da APS, que é o valor médio dos atributos essenciais e derivados e o do grau de afiliação. Valores iguais ou maiores a 6,6 foram considerados satisfatórios. Para o cálculo realiza-se um ajustamento dos escores apontados na escala de Likert original a uma escala entre zero e 10, para cada um dos atributos avaliados $^{13}$.

O presente estudo foi aprovado pelo Comitê de Ética em Pesquisas da Universidade Estadual de Montes Claros.

\section{Resultados}

A coleta de dados incluiu todas as crianças menores de cinco anos, da comunidade, totalizando 76 famílias. Não houve recusas ou perdas. Os principais cuidadores referidos para as crianças foram as mães $(96,1 \%)$. Das crianças selecionadas, $40(52,6 \%)$ eram do sexo feminino. A média de idade do grupo estudado foi de 25,0 meses (DP \pm 16,9 meses) e todas as crianças alocadas para o estudo possuíam irmãos, cuja média era de 2,1 (DP $\pm 2,3)$. Observou-se que 67 (88,2\%) dos entrevistados identificaram a ESF como fonte regular de atenção para o cuidado de saúde das crianças, enquanto nove cuidadores $(11,8 \%)$ consideraram outros serviços como fonte regular. A Tabela 1 consolida as principais características sociodemográficas do grupo estudado.
Tabela 1. Características sociodemográficas de crianças e famílias quilombolas. São Francisco (MG) - Comunidade de Buriti do meio, 2011.

\begin{tabular}{|c|c|c|}
\hline Variáveis & n (\%) & Média (DP) \\
\hline Idade das crianças em meses & & $25,0 \pm 16,9$ \\
\hline Idade das mães em anos & & $27,3 \pm 7,8$ \\
\hline Idade dos pais em anos & & $31,1 \pm 9,1$ \\
\hline Número de irmãos & & $2,1 \pm 2,3$ \\
\hline Número de pessoas na família & & $6,7 \pm 4,2$ \\
\hline Número de cômodos & & $5,4 \pm 1,9$ \\
\hline Tempo na comunidade (anos) & & $26,3 \pm 10,0$ \\
\hline \multicolumn{3}{|l|}{ Presença de água encanada } \\
\hline Não & $8(10,5)$ & \\
\hline Sim & $68(89,5)$ & \\
\hline \multicolumn{3}{|l|}{ Esgotamento sanitário } \\
\hline Sanitário com descarga & $49(64,5)$ & \\
\hline Sanitário sem descarga & $2(3,0)$ & \\
\hline Fossa rudimentar & $6(7,9)$ & \\
\hline Não tem & $19(25,0)$ & \\
\hline \multicolumn{3}{|l|}{ Tipo de construção } \\
\hline Tijolo & $52(68,4)$ & \\
\hline Outros & $24(31,6)$ & \\
\hline \multicolumn{3}{|l|}{ Cuidador principal } \\
\hline Mãe & $73(96,1)$ & \\
\hline Outros & $3(3,9)$ & \\
\hline \multicolumn{3}{|l|}{ Classe econômica ${ }^{*}$} \\
\hline Classe A & $0(0)$ & \\
\hline Classe B & $0(0)$ & \\
\hline Classe C & $18(25,0)$ & \\
\hline Classe D & $33(43,4)$ & \\
\hline Classe E & $24(31,6)$ & \\
\hline \multicolumn{3}{|l|}{ Escolaridade materna } \\
\hline Analfabeto & $2(2,6)$ & \\
\hline Fundamental incompleto & $54(71,1)$ & \\
\hline Fundamental completo & $18(23,7)$ & \\
\hline Médio completo & $2(2,6)$ & \\
\hline Médio incompleto & $0(0)$ & \\
\hline Não informado & $0(0)$ & \\
\hline \multicolumn{3}{|l|}{ Escolaridade paterna } \\
\hline Analfabeto & $13(17,1)$ & \\
\hline Fundamental incompleto & $49(64,5)$ & \\
\hline Fundamental completo & $3(3,9)$ & \\
\hline Médio completo & $5(6,6)$ & \\
\hline Médio incompleto & $0(0)$ & \\
\hline Não informado & $6(7,9)$ & \\
\hline
\end{tabular}

(*) Critério Brasil de Classificação Econômica

A Tabela 2 apresenta os escores dos atributos da APS, aferidos a partir da percepção dos cuidadores entrevistados, para a assistência à população infantil na comunidade estudada. Valores satisfatórios (escores $\geq 6,6$ ) foram registrados apenas para a subdimensão do acesso de 
Tabela 2. Média dos Escores dos atributos da APS, nos serviços identificados pelos cuidadores como fonte regular de atenção à saúde de crianças quilombolas. São Francisco (MG) - Comunidade de Buriti do meio, 2011.

\begin{tabular}{lc}
\hline \multicolumn{1}{c}{ Atributos da APS } & Média (DP) \\
\hline Acesso - Utilização & $9,1 \pm 1,7$ \\
Acesso - Acessibilidade & $4,8 \pm 2,2$ \\
Longitudinalidade & $6,1 \pm 1,8$ \\
Coordenação - Integralidade do cuidado & $5,6 \pm 3,2$ \\
Coordenação - Sistema de informação & $6,9 \pm 1,8$ \\
Integralidade - Serviços disponíveis & $6,3 \pm 2,0$ \\
Integralidade - Serviços prestados & $5,5 \pm 3,6$ \\
Orientação Familiar & $3,8 \pm 2,6$ \\
Orientação Comunitária & $5,8 \pm 1,9$ \\
Geral & $6,4 \pm 1,3$ \\
\hline
\end{tabular}

(*) Critério Brasil de Classificação Econômica primeiro contato - utilização e para a subdimensão coordenação - sistema de informação. O escore geral da APS, na percepção dos cuidadores, não alcançou o valor de mínimo para ser considerado como satisfatório.

\section{Discussão}

No presente estudo a avaliação dos serviços de saúde destinados à população quilombola revelou que quase todos os atributos da atenção primária não estão adequadamente presentes no processo assistencial. Em outras palavras, não existe aderência dos serviços ao novo modelo assistencial e perpetua-se a proposta de assistência pontual e curativista com centralidade nos aspectos biológicos do processo saúde-doença. Este resultado significa, por si mesmo, uma situação de não conformidade à proposta de reformulação do modelo assistencial. Entretanto, considerando as dificuldades socioeconômicas e a vulnerabilidade da comunidade avaliada, este resultado assume maior dimensão, pois retrata a perversa iniquidade que a estão submetidos os quilombolas da comunidade avaliada.

A vulnerabilidade e a fragilidade de comunidades rurais já têm sido destacadas pela literatu$\mathrm{ra}^{14}$. Na verdade, as condições de saúde de uma comunidade são fruto de uma complexa interação de fatores. O que se salienta, entretanto, com os resultados observados, é o não reconhecimento explícito por parte da comunidade estudada de aspectos diferenciais da atenção à saúde, que têm o potencial de produzir impacto positivo sobre os indicadores de saúde.

A escassez de estudos sobre o tema já foi apontada por outros autores ${ }^{15}$. Este aspecto destaca a importância de estudos que envolvam o recorte étnico/racial na assistência à saúde e, particularmente em relação às condições de vida da população negra e quilombola, salienta a vulnerabilidade de considerável contingente populacional.

As características da população estudada mostram que as crianças quilombolas vivem em condições socioeconômicas precárias, moram em locais desconfortáveis e suas famílias possuem parcos recursos de acesso aos bens e serviços em geral. Foram identificadas famílias cujos pais e mães são jovens, com baixa escolaridade, com número de filhos acima da média brasileira, residindo em casas pequenas, muitas delas sem estrutura adequada de saneamento ou conforto mínimo. A atividade produtiva do pai e da mãe das crianças são aquelas consideradas não qualificadas, reforçando o que a literatura já retratou sobre tais comunidades ${ }^{16}$. A maioria dos habitantes da comunidade quilombola avaliada dedicava-se à agricultura de subsistência.

É possível supor que, no cenário apresentado, as condições de saúde não sejam as melhores possíveis e, consequentemente, que estas necessidades sejam grandes, particularmente para as crianças. Os escores observados para os atributos da APS denotam, de modo global, um descompasso entre as ações de saúde desenvolvidas e as desejáveis neste cenário de iniquidade.

A identificação da ESF como serviço de referência para o cuidado de saúde das crianças é traduzida pelo escore acesso de primeiro contato-utilização. Neste estudo, a unidade foi referida para mais de $80 \%$ dos entrevistados. Trata-se de um índice superior ao apresentado em outros estudos que utilizaram o mesmo instrumento ${ }^{10,17}$. Se por um lado essa proporção traduz a legitimidade e o reconhecimento da unidade da ESF por parte da comunidade assistida, por outro, pode sinalizar tão somente que se trata do único acesso disponível à comunidade, que está a cerca de 17 quilômetros distante de algumas das residências da comunidade. Alguns estudos têm demonstrado que os estratos mais pobres utilizam, com mais frequência, os serviços de atenção primária como fonte regular de assistência à saúde ${ }^{12,18}$. Também se identificam entre os motivos que levam a população a escolher o local para acompanhamento de saúde, a proximidade da moradia e a qualidade do atendimento ${ }^{19}$. 
No presente estudo não se registrou o reconhecimento da população acerca da acessibilidade ao serviço de saúde, pois a subdimensão acesso de primeiro contato-acessibilidade apresentou escore insatisfatório. Este escore traduz a disponibilidade do serviço de saúde em relação à necessidade da população (consultas no mesmo dia, atendimento sem demora, agendamento fácil, etc.). Em síntese, apesar do atributo do primeiro contato, na subdimensão "utilização", ter obtido escore adequado, chama atenção que o escore da subdimensão "acessibilidade" mostrou-se insatisfatório, e isto demonstra que ainda existem barreiras que dificultam o acesso das crianças aos serviços de saúde. Esse fato também foi identificado em outros estu$\operatorname{dos}^{10,12}$. Os resultados deste estudo estão em consonância com os achados de Leão et al. ${ }^{10}$ e Van Stralen et al. ${ }^{12}$, que registram um modo de funcionamento das equipes da ESF que não se mostra disponível às necessidades da população.

Em relação à longitudinalidade, o escore registrado não foi considerado satisfatório. Esse escore é avaliado em 14 itens e traduz a existência do aporte regular de cuidados pela equipe de saúde e seu uso consistente ao longo do tempo, além do vínculo ou conhecimento dos profissionais de saúde com a comunidade. A presença adequada da longitudinalidade é um fator essencial para o sistema de saúde, pois este atributo tende a produzir diagnósticos e tratamentos mais precisos, além da redução de encaminhamento desnecessários que oneram os sistemas de saúde com a realização de procedimentos de maior complexidade. É possível que a inconstância da presença do médico e/ou a rotatividade dos profissionais de saúde justifiquem o resultado observado. A questão dos recursos humanos nas equipes de Saúde da Família é um evento complexo e que compromete toda a efetividade da estratégia ${ }^{20}$. As condições geográficas das equipes quilombolas se apresentam, nesse contexto, como pontos críticos e desfavoráveis à fixação dos profissionais.

Para a subdimensão da coordenação - sistema de informação registrou-se escore satisfatório. Essa subdimensão é avaliada por meio de três itens, que abordam o sistema de registro das informações do paciente (no caso, caderneta da criança e prontuário). Apesar de uma situação adequada em relação a essa subdimensão, a avaliação dos quesitos complementares da coordenação, relacionados à integração de cuidados, não se mostrou satisfatória. A subdimensão de integração de cuidados implica na capacidade de se garantir a continuidade da atenção na rede de serviços. Para existir coordenação do cuidado é ne- cessário que ocorra transferência de informações sobre os problemas dos pacientes, bem como da assistência recebida em outros pontos de atenção, possibilitando assim a continuidade do cuidado. Registra-se, portanto, que diante de uma necessidade explícita do paciente, a ESF não assume a postura de responsabilidade pelo seu deslocamento e assistência em outros locais, cabendo essa tarefa à família. Conclui-se, então que a rede assistencial se mostra frágil em relação ao trânsito dos pacientes em diferentes níveis de atenção.

Para as demais dimensões avaliadas pelo PCATool, os escores avaliados não se mostraram satisfatórios. Assim, a integralidade da assistência, a orientação familiar e a orientação comunitária não são reconhecidas pela comunidade assistida. O baixo escore da integralidade denota a persistência da dicotomia (que sempre existiu no país), entre as ações médico-assistenciais e as ações preventivas ${ }^{21}$. O fato demonstra a necessidade de que sejam disponibilizados mais serviços para atenderem às necessidades básicas de saúde da população, incluindo insumos como medicamentos básicos e vacinas.

Registra-se também a necessidade de uma melhor qualificação dos profissionais em relação aos cuidados no manejo das condições mais comuns e de grande impacto na saúde geral das famílias e da comunidade. Pesquisa realizada com os ACS que atuam nesta comunidade demonstrou, por exemplo, que a falta de capacitação é percebida pelos próprios agentes que são membros da comunidade quilombola ${ }^{22}$. Os estudos têm reforçado que dentre os problemas de saúde e agravos observados em populações quilombolas, estão o consumo de álcool, os problemas mentais, a doença falciforme e a hipertensão ${ }^{16,22}$. Entre as crianças, destaca-se que a taxa de mortalidade tende a ser mais elevada em comparação às populações urbanas e às de outras comunidades rurais do Brasil ${ }^{23}$. Assim, torna-se necessário o aumento do escopo de atuação dos serviços de saúde, uma vez que esta medida pode contribuir para melhores indicadores de saúde.

Os escores da orientação familiar e da orientação comunitária inadequados indicam que o modelo assistencial ainda não integra o indivíduo à sua família e não o reconhece como pertencente a uma comunidade específica. Algumas características deveriam estar relacionadas com a melhor efetividade desses atributos na ESF, como as ações de visita domiciliar. Essa ação, apesar de simples, deve ser realizada por todos os profissionais, pois facilita a vigilância à saúde e o acompanhamento das famílias da comuni- 
dade. Neste estudo a presença dessas ações, não foi suficiente para alcançar escore adequado.

A análise conjunta dos atributos - escore geral - registra que os serviços fontes de atenção à saúde das crianças quilombolas estão ainda aquém do ideal. Resultados similares foram observados em outros estudos, salientando que a ESF ainda necessita maior refinamento de suas ações para promover uma APS em consonância com seus atributos ${ }^{10}$. O que representa um aspecto particularmente grave em relação aos resultados observados é o registro de escores baixos em uma comunidade tão sofrida e cronicamente esquecida. O rótulo de "PSF quilombola" não diferencia o resultado final do trabalho, pois não existe um reconhecimento das necessidades de saúde da população, ações de orientação familiar ou comunitária.

A APS de qualidade é uma estratégia efetiva na busca de maior promoção da saúde, prevenção de doenças e tem potencial para melhorar o estado de saúde das pessoas com maior satisfação dos cuidadores ${ }^{8}$. Equipes de Saúde da Família adequadamente formadas e comprometidas podem desenvolver um papel fundamental no enfrentamento às iniquidades de populações marginalizadas, incluindo-se as comunidades quilombolas.

No presente estudo os resultados não permitem assegurar que essa população tem recebido a melhor assistência possível. Assim, intervenções na área da gestão com melhoria da infraestrutura, reorganização das ações e capacitação dos profissionais de saúde devem ser prioritárias para a população estudada. A percepção de um modelo ainda não alcançado também foi re- gistrada na avaliação da atenção à saúde infantil da ESF por outros autores. Em estudo conduzido no sul de Minas Gerais, os autores destacam que a atuação da equipe da ESF na atenção à saúde das crianças ainda está distante da proposta de reorientação do modelo assistencial, o que compromete as atividades de prevenção de doenças e promoção da saúde ${ }^{24}$.

Finalmente, destaca-se que os resultados devem ser interpretados considerando algumas limitações. Uma delas é a carência de estudos nacionais sobre o tema, principalmente entre populações quilombolas, o que aponta para a necessidade de novas avaliações similares. $\mathrm{O}$ fato de a avaliação retratar a percepção de uma única comunidade também representa um limitante à generalização dos dados. Entretanto deve-se destacar que a mesma realidade socioeconômica e o mesmo histórico de segregação e abandono estão presentes em muitas comunidades do norte e nordeste do País, o que enseja, minimamente, uma reflexão mais profunda sobre questões de acesso à saúde e a equidade para tais comunidades. É preciso salientar ainda que a percepção registrada não incluiu todos os atores sociais, e as perspectivas dos gestores e prestadores de serviços também devem ser conhecidas, para um melhor enfretamento da situação. Enfim, para a consolidação do modelo assistencial proposto pela APS são necessários estudos contínuos, que possibilitem avaliar a evolução dos atributos nos serviços de saúde, em diferentes perspectivas. Para comunidades que sempre foram alijadas dos programas de inclusão e desenvolvimento social essa necessidade deve ser um compromisso dos setores acadêmicos.

\section{Colaboradores}

AS Marques, DA Freitas, CDA Leão, SKM Oliveira, MM Pereira e AP Caldeira participaram igualmente de todas as etapas de elaboração do artigo. 


\section{Referências}

1. Brasil. Ministério da Saúde (MS). Secretaria-Executiva. Subsecretaria de Planejamento e Orçamento. A saúde da população negra e o SUS: ações afirmativas para avançar na eqüidade. Brasília: Editora do Ministério da Saúde; 2005.

2. Senna MCM. Eqüidade e política de saúde: algumas reflexões sobre o Programa Saúde da Família. Cad Saude Publica 2002; 18(Supl.):203-211.

3. Coelho IB. Democracia sem equidade: um balanço da reforma sanitária e dos dezenove anos de implantação do Sistema Único de Saúde no Brasil. Cien Saude Colet 2010; 15(1):171-183.

4. Escorel S, Giovanella L, Mendonça MHM, Mônica de Castro Maia Senna MCM. O Programa de Saúde da Família e a construção de um novo modelo para a atenção básica no Brasil. Rev Panam Salud Publica 2007; 21(2-3):164-176.

5. Macinko J, Guanais FC, Fátima M, Souza M, Souza M. Evaluation of the impact of the Family Health Program on infant mortality in Brazil, 1990-2002.J Epidemiol Community Health 2006; 60(1):13-19.

6. Fernandes VBL, Caldeira AP, Faria AA, Rodrigues Neto JF. Internações sensíveis na atenção primária como indicador de avaliação da Estratégia Saúde da Família. Rev Saude Publica 2009; 43(6):928-936.

7. Kalckmann S, Santos CG, Batista LE, Cruz VM. Racismo Institucional: um desafio para a eqüidade no SUS? Saúde Soc 2007; 16(2):146-155.

8. Starfield B. Atenção Primária: equilíbrio entre necessidades de saúde, serviços e tecnologia. Brasília: UNESCO, Ministério da Saúde (MS); 2002.

9. Caminal J, Starfield B, Sánchez E, Casanova C, Morales $\mathrm{M}$. The role of primary care in preventing ambulatory care sensitive conditions. Eur J Public Health 2004; 14(3):246-251.

10. Leão CDA, Caldeira AP, Oliveira MMC. Atributos da atenção primária na assistência à saúde da criança: avaliação dos cuidadores. Rev Bras Saude Mater Infant 2011; 11(3):323-334.

11. Macinko J, Almeida C, Oliveira ES, Sá PK, Organization and delivery of primary health care services in Petrópolis, Brazil. Int J Health Plann Manage 2004; 19(4):303-317.

12. Van Stralen CJ, Belisário AS, Van Stralen TBS, Lima, AMD, Massote AW, Oliveira CL. Percepção dos usuários e profissionais de saúde sobre atenção básica: comparação entre unidades com e sem saúde da família na Região Centro-Oeste do Brasil. Cad Saude Publica 2008; 24(Supl. 1):148-158.

13. Brasil. Ministério da Saúde (MS). Secretaria de Atenção em Saúde. Departamento de Atenção Básica. Manual do instrumento de avaliação da atenção primária à saúde: primary care assessment tool - PCAtool - Brasil. Brasília: MS; 2010.
14. Pignatti MG, Castro SP. A fragilidade/resistência da vida humana em comunidades rurais do Pantanal Mato-Grossense (MT, Brasil). Cien Saude Colet 2010; 15(Supl. 2):3221-3332.

15. Freitas DA, Diaz Caballero A, Marques AS, Vergara Hernández CI, Antunes SLNO. Saúde e comunidades quilombolas: uma revisão da literatura. Rev CEFAC 2011; 13(5):937-943.

16. Silva, JAN. Condições sanitárias e de saúde em Caiana dos Crioulos, uma comunidade Quilombola do Estado da Paraíba. Saude soc 2007; 16(2):111124.

17. Ribeiro LCC. Acolhimento as crianças na atenção primaria à saúde: um estudo sobre a postura dos profissionais das equipes de saúde da família [dissertação]. Belo Horizonte: Universidade Federal de Minas Gerais; 2009.

18. Silva NN, Pedroso LC, Puccini RF, Furlani WJ. Desigualdades sociais e uso de serviços de saúde: evidências de análise estratificada. Rev Saude Publica 2000; 34(1):44-49.

19. Ramos DD, Lima MADS. Acesso e acolhimento aos usuários em uma unidade de saúde de Porto Alegre, Rio Grande do Sul, Brasil. Cad Saude Publica 2003; 19(1):27-34.

20. Gil CRR. Formação de recursos humanos em saúde da família: paradoxos e perspectivas. Cad Saude Publica 2005; 21(2):490-498.

21. Campos CEA. O desafio da integralidade segundo as perspectivas da vigilância da saúde e da saúde da família. Cien Saude Colet 2003; 8(2):569-584.

22. Freitas DA, Silveira JCS, Ferreira LA, Zucchi P, Marques AS. Mulheres quilombolas: profissionais na estratégia de saúde da família. Rev Espaço para Saúde 2011; 12(2):56-62.

23. Guerrero AFH, Silva DO, Toledo LM, Guerrero JCH, Teixeira P. Mortalidade infantil em remanescentes de quilombos do Munícipio de Santarém Pará, Brasil. Saude Soc 2007; 16(2):103-110.

24. Costa GD, Cotta RMM, Reis JR, Ferreira MLSM, Reis RS, Franceschini SCC. Avaliação da atenção à saúde da criança no contexto da Saúde da Família no município de Teixeiras, Minas Gerais (MG, Brasil). Cien Saude Colet 2011; 16(7):3229-3240.

Artigo apresentado em 15/03/2013

Aprovado em 31/03/2013

Versão final apresentada em 02/04/2013 
